\title{
Non-adiabatic seismic study of the thin convective envelope of $\delta$ Scuti stars
}

\author{
M.-A. Dupret, A. Grigahcène, R. Garrido \\ Instituto de Astrofísica de Andalucía-CSIC, Apartado 3004, 18080 \\ Granada, Spain \\ J. Montalban, M. Gabriel, R. Scuflaire \\ Institut d'Astrophysique et de Géophysique, Université de Liège, allée \\ du Six Août 17, B-4000 Liège, Belgium
}

\begin{abstract}
For $\delta$ Sct stars, the theoretical predictions of a non-adiabatic pulsation code are very dependent on the characteristics of the thin convective envelope of the models (Balona \& Evers 1999). The treatment of the non-adiabatic interaction between convection and pulsation also has a significant impact on the results, particularly near the red edge of the instability strip. The non-adiabatic theoretical predictions can be tested upon observations by comparing them to the amplitude ratios and phase differences as observed in different color passbands (Dupret et al. 2003). In the first part of this paper, we compare the results obtained by adopting different treatments of convection in the interior and atmosphere models: mixing-length theory (MLT) and full spectrum of turbulence (FST) (Canuto et al. 1996, CGM). In the second part, we examine the problem of the interaction between convection and pulsation and compare the mode stability obtained with and without including time-dependent convection in our non-adiabatic code.
\end{abstract}

\section{Sensitivity of non-adiabatic pulsation observables on stationary convection models}

\subsection{Convection in interior and atmosphere stellar models}

$\delta$ Sct stars (A-F type stars) have a thin, inefficient superficial convective zone extending up to the photosphere. In this paper, we compare the results obtained with different convection models in the interior and in the atmosphere of the star, following the MLT approach with $\alpha=l / H_{\mathrm{p}}=0.5, \alpha=1.25$ or the FST approach. The interior models were computed using the evolutionary codes ATON3.0 (FST and MLT) and CLES (MLT). For the atmosphere models, we have used the ATLAS9 MLT models with $\alpha=1.25$ (Kurucz 1993) and $\alpha=0.5$ (Heiter et al. 2002); and the ATLAS9 FST models (CGM) (Heiter et al. 2002). All the models of this section are with $T_{\text {eff }}=7400 \mathrm{~K}$ and $\log g=4.0$. 

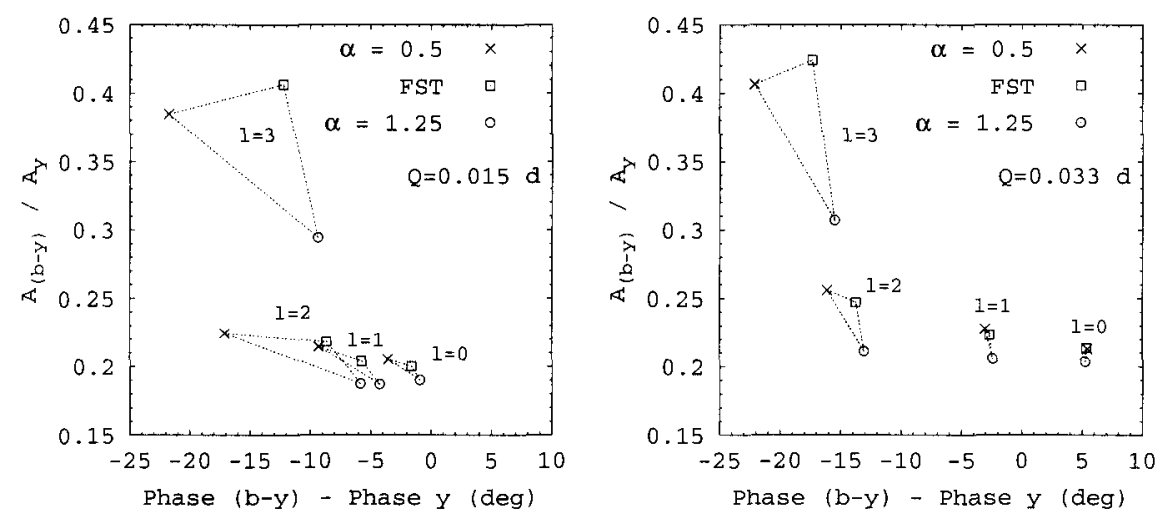

Figure 1. Phase-amplitude diagrams obtained for different models of convection, for modes of degree $\ell=0,1,2,3$. The constant of pulsation is $Q=0.015 \mathrm{~d}$ in the left panel and $Q=0.033 \mathrm{~d}$ in the right panel.

\subsection{Results}

We performed non-adiabatic computations for all these models using the nonadiabatic code MAD (Dupret et al. 2002). The phase-lags and the effective temperature variations predicted by the non-adiabatic pulsation code appear to be very sensitive to the details of the thin convective zone. Theoretical multi-color photometric amplitude ratios and phase differences depend on these non-adiabatic results and on the monochromatic flux and limb-darkening of the atmosphere models (Dupret et al. 2003). The limb-darkening coefficient for the FST models have been taken from Barban et al. (2003). In Fig. 1, we show the results obtained in phase-amplitude diagrams for Strömgren filters.

\section{Non-adiabatic interaction between convection and pulsation in $\delta$ Scuti stars}

\subsection{Non-adiabatic convection theory of Gabriel (1996)}

Turbulence is usually described in terms of mean and fluctuation equations. In the theory of Gabriel (1996), the fluctuation equations of continuity, of motion and of energy conservation have respectively the following linearized form.

$$
\begin{gathered}
\nabla \cdot \vec{V}=0 \\
\bar{\rho} \frac{d \vec{V}}{d t}=\frac{\Delta \rho}{\rho} \nabla \bar{P}-\nabla \Delta P-\frac{8 \rho \vec{V}}{3 \tau_{\mathrm{c}}}-\rho \vec{V} \cdot \nabla \vec{u} \\
\frac{\Delta(\rho T)}{\overline{\rho T}} \frac{d \bar{s}}{d t}+\frac{d \Delta s}{d t}+\vec{V} \cdot \nabla \bar{s}=-\frac{\Gamma^{-1}+1}{\tau_{\mathrm{c}}} \Delta s
\end{gathered}
$$


where $\tau_{\mathrm{c}}$ is the life time of the convective elements and $\Gamma$ is the convective efficiency.

Stationary solutions of the above equations lead to the classical MLT theory of Böhm-Vitense. The Lagrangian perturbation of the mean equations gives the linear pulsation equations, where unknown terms such as the perturbation of the convective flux appear:

$$
\delta \vec{F}_{\mathrm{c}}=\overrightarrow{\vec{F}}_{\mathrm{c}}\left(\frac{\delta c_{p}}{c_{p}}+\frac{\delta \rho}{\bar{\rho}}+\frac{\delta T}{\bar{T}}\right)+c_{p} \bar{\rho} \bar{T}(\overrightarrow{\delta \Delta S \vec{V}}+\overrightarrow{\Delta S \delta \vec{V}}) .
$$

The unknown correlation terms of Eq. (4) can be obtained from the fluctuation equations. More precisely, we perturb Eqs (1) to (3) and search for solutions of the form $\delta(\Delta X)=\delta(\Delta X)_{\vec{k}} e^{i \vec{k} \cdot \vec{r}} e^{i \sigma t}$. Then we integrate these particular solutions over all values of $k_{\theta}$ and $k_{\phi}$ such that $k_{\theta}^{2}+k_{\phi}^{2}=A k_{r}^{2}(A=1 / 2$ for an isotropic turbulence). Finally, the perturbed convective flux takes the following form:

$$
\delta \vec{F}_{\mathrm{c}}=\delta F_{\mathrm{cr}}(r) Y_{l}^{m}(\theta, \phi) \vec{e}_{r}+\delta F_{\mathrm{ch}}(r)\left(r \nabla_{h} Y_{l}^{m}(\theta, \phi)\right)
$$

and the problem is naturally separated in spherical harmonics. $\delta F_{\mathrm{cr}}(r)$ and $\delta F_{\mathrm{ch}}(r)$ are related to the perturbed mean quantities by first order differential equations.

The main source of uncertainty of any MLT theory of convection-pulsation interaction is in the way to perturb the mixing-length $l$. Gough (1977) proposed to perturb only the pressure scale height: $\delta l / l=\delta H_{\mathrm{p}} / H_{\mathrm{p}}$. However, it can be expected that the perturbation of the mixing-length becomes negligible when the life-time of the convective elements is much longer than the period of pulsation, this can be reproduced for example by adopting:

$$
\delta l / l=1 /\left(1+\left(\sigma \tau_{\mathrm{c}}\right)^{2}\right) \delta H_{\mathrm{p}} / H_{\mathrm{p}} .
$$

\subsection{Red edge of the $\delta$ Scuti instability strip}

A very good confirmation of the quality of our time-dependent convection treatment is that it is able to reproduce the red edge of the $\delta$ Sct instability strip, contrary to models with frozen convection. In Fig. 2, we give the evolution of the dimensionless frequencies $\left(\omega=\sigma t_{\mathrm{dyn}}\right)$ of $\ell=2$ modes as a function of effective temperature and age, for $1.8 \mathrm{M}_{\odot}$ models with overshooting $\alpha_{\mathrm{ov}}=0.2$ and mixing-length parameter $\alpha=1.5$. The right panel corresponds to the results obtained with time-dependent convection in our non-adiabatic code, the left panel is without.

\section{Conclusions}

The theoretical non-adiabatic amplitudes and phases of effective temperature variations, as well as the monochromatic flux and limb-darkening derivatives are very sensitive to the stationary convection models in the thin convective envelope of $\delta$ Sct stars. This has a significant impact on the photometric amplitude ratios and phase differences as observed in different color passbands. A confrontation with accurate observations could thus allow discrimination between these different convection models. 

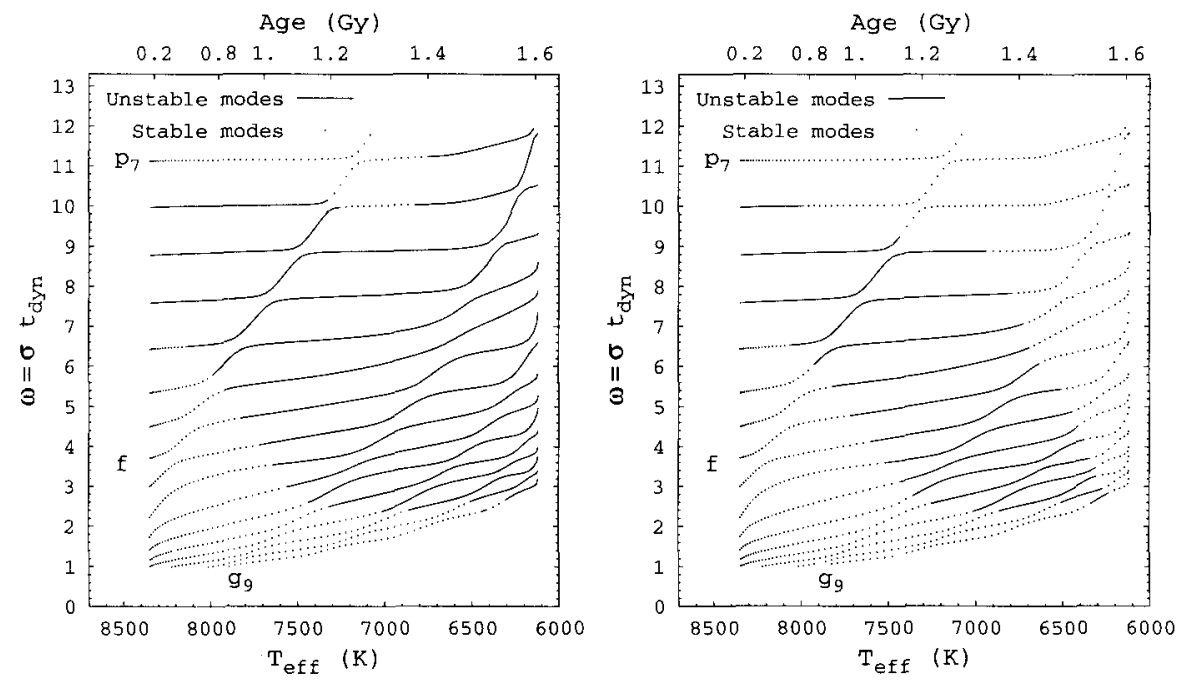

Figure 2. Stable and unstable modes obtained with (right) and without (left) including time-dependent convection in the models.

Our non-adiabatic time-dependent treatment of the interaction between convection and pulsation allows us to predict a red edge of the $\delta$ Sct instability strip in agreement with observations for radial as well as for non-radial modes. This red edge cannot be obtained with frozen convection models.

Acknowledgments. Part of this work was supported through a European Community Marie Curie Fellowship.

\section{References}

Balona, L.A., Evers, E.A. 1999, MNRAS, 302, 349

Barban, C., Goupil, M.J., Van't Veer-Menneret, C., Garrido, R., Kupka, F., Heiter, U. 2003, A\&A, 405, 1095

Canuto, V.M., Goldman, I., Mazzitelli, I. 1996, ApJ, 473, 550 (CGM)

Dupret, M.-A., De Ridder, J., Neuforge, C., Aerts, C., Scuflaire, R. 2002, A\&A, 385,563

Dupret, M.-A., De Ridder, J., De Cat, P., Aerts, C., Scuflaire, R., Noels, A., Thoul, A. 2003, A\&A, 398, 677

Gabriel, M. 1996, Bull. Astr. Soc. India, 24, 233

Gough, D.O. 1977, ApJ, 214, 196

Heiter, U., Kupka, F., van't Veer-Menneret, C., Barban, C., et al. 2002, A\&A, 392,619

Kurucz, R.L. 1993, ATLAS9 Stellar Atmosphere programs and $2 \mathrm{~km} / \mathrm{s}$ grids. Kurucz CDROM No 13 